\title{
Plank's Radiation Law as a Consequence of Nonequilibrium Thermodynamics
}

\author{
V.A. Etkin ${ }^{1 *}$ \\ ${ }^{1}$ Togliatti State University, Research Center., Advisor to the Vice-Rector for Science \\ E-mail: ${ }^{1}$ etkin.v@mail.ru
}

Received 26 August 2019, Revised 30 October 2019, Accepted 2 November 2019

\begin{abstract}
It is shown that the Planck radiation law can be deduced from the first principles of nonequilibrium thermodynamics without using the Planck hypothesis and other quantum-mechanical considerations. The conclusion is based on the concept of radiation as a process in which the frequency plays the role of a wave flow, and the oscillator oscillators have an unlimited number of harmonics. In this case, a single wave becomes discrete, discrete both in time and in space, and the Planck constant acquires the meaning of an averaged specific impulse of waves emitted by a completely black body. Moreover, the de Broglie relation follows as a consequence of the law of radiation and reflects the particle-like properties of the wave. The proposed approach removes a number of contradictions of quantum theory with classical physics.
\end{abstract}

Keywords: Radiation laws, Planck hypothesis, nonequilibrium thermodynamics, wave as a quantum, wave-particle dualism, quantum theory.

\section{Introduction}

One of the main reasons that gave rise to the quantum relativistic revolution at the turn of the nineteenth and twentieth centuries was the lack of a satisfactory explanation for the experimentally established distribution of the spectral density of radiation of bodies [1]. By this time it was already known that the radiation of bodies does not cease with the onset of thermal equilibrium, and the stationary state of the emitter is incompatible with equilibrium and is a consequence of the equality of the fluxes of radiated and absorbed energy. However, a radiation theory that could take this circumstance into account did not exist at that time. Therefore, M. Planck, like his predecessor Rayleigh, used the Boltzmann concept of radiation in an imaginary cavity of an absolutely black body as a kind of substance in thermal equilibrium with an emitter [2]. With this approach, radiation was attributed a certain temperature $\mathrm{T}$ and entropy $\mathrm{S}$, i.e., parameters inherent to the emitter. The focus of Planck was the expression for the second derivative of the entropy of an ideal gas $\mathrm{S}$ with respect to its energy $\mathrm{U}$. In the shortwavelength region (where Wien's law is valid), the derivative $\partial^{2} \mathrm{~S} / \partial \mathrm{U}^{2}$ would and inversely proportional to $\mathrm{U} 2$, while in the long-wavelength region - the first degree of energy U. Planck constructed the quantity $\partial^{2} \mathrm{~S} / \partial \mathrm{U}^{2}=\mathrm{a} / \mathrm{U}(\mathrm{U}$ $+b$ ), which gives their simplest generalization, and thereby found a "successful interpolation formula", which satisfies experimental data in the entire frequency range [2].

However, for the subsequent substantiation of this procedure, M. Planck had to resort to a number of postulates that contradict classical physics. The main one was the assumption that the atoms of matter as oscillators can only be in certain discrete energy states with energies $\varepsilon_{\mathrm{n}}$ $=n h v$ proportional to the frequency $v$, where $n=1,2, \ldots \infty$ are quantum numbers that form a natural series, and emission and absorption radiation occurs in indivisible portions with energy $\varepsilon_{0}=h v$. According to this relation, the energy of a radiation quantum was determined exclusively by its frequency and did not depend either on the wave amplitude or on the temperature and other properties of the emitter, which contradicted the theory of oscillations. And although A. Poincare in a number of articles proved in a very rigorous and general way the impossibility of obtaining the law of equilibrium radiation without the quantum hypothesis [3], Planck himself considered the problem of thermal radiation unresolved until the end of his life [4] .

The situation changed significantly only with the advent of the thermodynamics of stationary irreversible processes (TIP), which operates with the concept of energy flows and their carriers $[5,6]$. It became possible to describe the process of non-equilibrium radiation without resorting to model ideas far from reality and to postulates alien to classical physics. The essence of this approach is summarized below.

\section{The Specifics of the Radiation Process from the Standpoint of TIP}

The principal difference of the proposed approach is the consideration of radiation as an irreversible process of radiant energy exchange between the emitter and its environment. This process is by no means reducible to heat transfer, which occupies a very insignificant part of the frequency range, and is accompanied by the completion of useful work "against equilibrium". This is evidenced by the phenomena of photosynthesis, photoelectric effects, photochemical, photonuclear, etc. The stationarity of the state of the emitter is due in this case to the equality of the 
fluxes of the absorbed $\mathbf{j}$ ' and the "reradiated" energy $\mathbf{j}$ ", and not their disappearance.

This required the generalization of consumer goods to the processes of converting ordered forms of energy into the internal energy of the emitter $U$ and vice versa. Such a generalization was carried out by us in the doctoral dissertation [7] and monographs [8,9]. Thermokinetics as a local nonequilibrium thermodynamics and energodynamics as its extension to any forms of energy study the kinetics of real processes of energy transfer and conversion, without excluding any (reversible or irreversible) part of them from consideration. To this end, they are based not on the expression for "entropy production" $d S_{i} / d t>0$, but on the more general law of energy conservation $d U / d t=0$. Due to this, they do not need the local equilibrium hypothesis and give consistently thermodynamic (not based on statistical considerations mechanical and quantum-mechanical nature) justification of all provisions of consumer goods. This served as the basis for the recommendation of "thermokinetics" as a textbook for Russian technical universities (1999) and the award of "energodynamics" with the Leibniz Medal (2009) of the European Academy of Natural Sciences.

From the standpoint of these theories, the flow of energy $\mathbf{j}_{\mathrm{r}}$ ' supplying the emitter excites in it the oscillatory process of any of its structural elements. This process is illustrated by Figure 1, from which it follows that a wave of an arbitrary physical quantity $\Theta$ (in this case, mass $M$ ) is formed by displacing some of its quantity $\Theta^{\prime}=\Theta^{\prime \prime}$ from a position with the radius vector $\mathbf{r}^{\prime}$ to position $\mathbf{r}^{\prime \prime}$ by a distance $\Delta \mathbf{r}=\mathbf{r}^{\prime \prime}-\mathbf{r}^{\prime}$, equal to the half-wavelength $\lambda / 2$. This shift is carried out over a half-period of oscillation with a frequency $v$ and takes place over a time $\Delta t=2 / v$, that is, it proceeds with an average speed $\mathbf{c}=\Delta \mathbf{r} / \Delta t=\lambda v$ numerically equal to the speed of light propagation in a given medium. If we take $|\Delta \mathbf{r}|$ for the amplitude $A_{v}$ of the longitudinal wave generated in this case with a frequency $v$, then the density $\rho_{v}$ of it's kinetic energy $\rho c^{2} / 2$ is expressed by the relation known from wave theory [10]:

$\rho_{v}=\rho A_{v}^{2} v^{2} / 2,\left(\mathrm{~J} \mathrm{~m}^{-3}\right)$

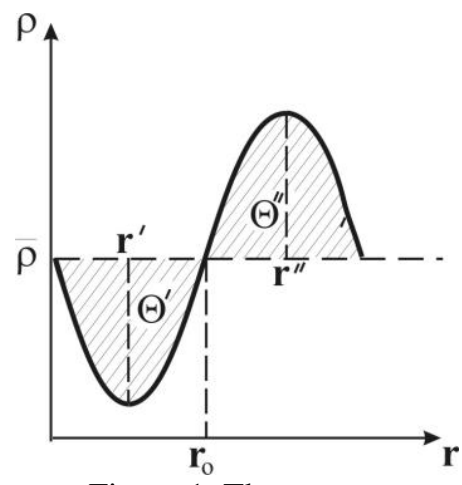

Figure 1. The wave.

Now, as usual, we represent the total derivative $d \rho_{v} / d t$ of the wave energy density (1) as the sum of the convective $(\mathbf{c} \cdot \nabla) \rho_{v}$ and local $\left(\partial \rho_{v} / \partial t\right)_{\mathrm{r}}$ component:

$d \rho_{v} / d t=\left(\partial \rho_{v} / \partial t\right)_{r}+\rho A_{\mathrm{B}} v \mathbf{c} \cdot \nabla\left(A_{v} v\right), \mathrm{W} \mathrm{m}{ }^{-3}$

The first term on the right-hand side of (2) characterizes the power of radiation absorbed by a unit volume body at a frequency $v$, the second is the flux of radiant energy transmitted by the emitter to the same environment. According to the TIP, this flow can be represented as the product of the flux density of the carrier of radiant energy $\mathbf{j}_{r}$ $=\rho \mathbf{c} A_{v} v$ and the thermodynamic force $\mathbf{X}_{r}=-\nabla\left(A_{v} v\right)$, expressed by the negative gradient of $\psi_{i}=A_{\nu} v$, which we called the "amplitude-frequency" wave potential [11]. In this case, the law of radiant energy exchange can be written in the form adopted in the theory of heat transfer [12] and physical kinetics for heat conduction, electrical conductivity, diffusion, etc. [13]:

$\mathbf{j}_{r}=L_{r} \mathbf{X}_{r}$

where $L_{r}$ is the proportionality coefficient, which generally depends on the state of the conducting medium and in this respect is similar to the coefficients of heat conductivity, electrical conductivity, diffusion, etc.

According to (2), in the stationary state of the emitter $\left(d \rho_{v} / d t=0\right)$, the power of the oscillatory process in the emitter at any frequency $\left(\partial \rho_{v} / \partial t\right)_{r}$ is equal to the flux of radiated or absorbed energy $\mathbf{j}_{r} \cdot \mathbf{X}_{r}$. It is easy to see that in this expression the frequency $v$ plays the role of the spectral wave flux $J_{v}=v$, i.e., the number of traveling single waves excited by the radiator in the environment per unit time. Thus, the frequency $v$ from the point of TIP acquires the meaning of the function of the radiation process, and not the function of the state of the emitter as a set of oscillators. It is quite natural that the flux of radiant energy in this case turns out to be proportional to the frequency as a wave flux.

This is the peculiarity of considering radiation from the standpoint of TIP as compared with equilibrium thermodynamics, where the frequency $v$ determined only the number of standing waves $n_{v}=d N_{v} / d V$, arising in the unit volume of the cavity of the blackbody, i.e., it was a function of the state of radiation as an oscillating substance. This made it difficult to understand the specifics of the radiation process. Now it is possible to consider the emitter itself as an object of study. If, moreover, we take into account that each of its oscillators, in addition to the fundamental vibration frequency vo, has $\mathrm{n}$ harmonics corresponding to the doubled, tripled, etc. frequency $v_{n}=$ $n v_{\mathrm{o}}(n=1,2,3$, etc. $)$, then the vibrational energy of each of such an oscillator en will appear as the sum of the energies $\varepsilon_{v}$ of all it's harmonics $n$ :

$\varepsilon_{n}=\Sigma_{n} \varepsilon_{v}(\mathbf{j})$

Formally, this expression differs from the Planck expression $\varepsilon_{n}=n \varepsilon_{o}$, only in that in it the abstract quantum numbers of the natural series $\mathrm{n}$ are replaced by harmonics characteristic of any oscillator. However, in this case, the oscillator energy is no longer assumed to consist of $n$ equal and indivisible parts (quanta) with the same energy $\varepsilon_{o}=h v$, proportional to the frequency $v$. Now the density of the radiation energy flux $\rho_{r}=d \mathbf{j}_{r} / d t$ is naturally determined by the integral of the average value of the oscillator energy $\left\langle\varepsilon_{n}\right\rangle$ by their total number $N$ per unit volume of the emitter:

$\rho_{r}=\int\left\langle\varepsilon_{n}\right\rangle d N, \quad \mathrm{~J} \mathrm{~m}^{-3}$

This makes it necessary in both cases to average the energy of the oscillators over a number of natural numbers $n$ in the entire frequency range $0<v<\infty$. Planck found that for blackbody, this problem can be solved by expanding 
$\exp \left(-\varepsilon_{v} / k T\right)$ in a series in $\mathrm{n}$ with the subsequent approximation of this series by the expression

$\left\langle\varepsilon_{n}\right\rangle=\varepsilon_{v} /\left[\exp \left(\varepsilon_{v} / k T\right)-1\right]$

The number $N$ of oscillators per unit volume depends on the frequency. However, it can be expressed in terms of the spectral density of oscillators oscillating with a frequency $v$, the well-known expression $n_{v}=d N / d v=$ $8 \pi v^{2} / c^{3}$. Since each such oscillation is accompanied by modulation of the traveling wave in the environment, the radiation density $\rho r$ is determined by integration over the entire frequency range of the radiation fluxes $\left\langle\varepsilon_{n}\right\rangle n_{v}$ of oscillators oscillating at a frequency $v$ :

$\rho_{r}=\int\left\langle\varepsilon_{n}\right\rangle n_{v} d v=\int\left(8 \pi h v^{2} \varepsilon_{v} / c^{3}\right) /\left[\exp \left(\varepsilon_{v} / k T\right)-1\right] d v$

Substitution of the Planck relation $\varepsilon_{v}=h v$ into this expression directly leads to the distribution law of the spectral radiation density $\rho_{v}=d \rho_{r} / d v=\left\langle\varepsilon_{n}\right\rangle n_{v}$, known as the Planck radiation law [2]:

$$
\rho_{v}=\left(8 \pi h v^{3} / c^{3}\right) /[\exp (h v / k T)-1]
$$

Thus, the radiation law (7) can be obtained in the classical way, without involving the hypotheses of quanta, but only assuming the distribution (6) over a number of natural numbers $\mathrm{n}$ obeying Maxwell-Boltzmann statistics. Moreover, the quantum hypothesis no longer lies at its foundation, and the fact that the flux of radiant energy $\mathbf{j}_{r}$ is proportional to the flux of waves $\mathbf{j}_{v}$ with averaged energy $\left\langle\varepsilon_{n}\right\rangle$ is not in doubt. In this case, the proportionality coefficient $h$ can be considered as a quantity subject to experimental determination. For blackbody, it can be found, for example, from the Stefan - Boltzmann law $\mathbf{j}_{r}=\sigma_{r} T^{4}(\mathrm{~W}$ $\left.\mathrm{m}^{-2}\right)$.

To verify this, it is first necessary to give expressions (7) and (8) a dimension corresponding to this law. For this, we pass in (8) from $\rho v$ to the spectral flux density of the radiant energy $j_{r}=\rho_{v} c\left(\mathrm{~J} \mathrm{~m}^{-2}\right)$. Then the modulus of the total (integral) flux of radiant energy $\mathbf{j}_{r}$ will get the meaning of the so-called "energy luminosity":

$\mathbf{j}_{r}=\int_{j_{r}} d v=\left(8 \pi / c^{2}\right) \int \varepsilon_{v} v^{2}\left[\exp \left(\varepsilon_{v} / k T\right)-1\right]^{-1} d v$

If we now denote $\exp \left(\varepsilon_{v} / k T\right)$ by $\mathrm{e}^{x}$, where $x=\varepsilon_{v} / k T=$ $h v / k T$, then expressions (7) and (8) can be reduced to

$\mathbf{j}_{r}=\left(8 \pi k^{4} / c^{2} h^{3}\right) \int x^{3}\left(e^{x}-1\right)^{-1} d x$

Given that the integral $\int x^{3}\left(e^{x}-1\right)^{-1} d x$ in the interval $0<x<1$, corresponding to the frequency range $0<v<\infty$, has the exact value $15 / \pi^{2}$, and comparing expression (10) with the experimental value $\sigma_{r}=5.67 \cdot 10^{-8}\left(\mathrm{~W} \mathrm{~m}^{-2} \mathrm{~K}^{-4}\right)$, we find for $k$ $=1.38 \cdot 10^{-23}\left(\mathrm{~J} \mathrm{~K}^{-1}\right)$ that the coefficient $h$ is the Planck constant $h=6.626 \cdot 10^{-34} \mathrm{~J}$ s Thus, this constant acquire the simple meaning of the averaged impulse of a traveling wave modeled by the blackbody in the surrounding luminiferous medium.

We now pay attention to the fact that, according to (8), ) $\varepsilon_{v}=h v$. Comparing this expression with the traveling wave momentum $p_{v} c=p_{v} \lambda v$, we immediately arrive at the de Broglie relation [14]:

$\lambda=h / p_{v}$
This relation was obtained without any hypotheses of a quantum-mechanical nature and reflects the presence of both a traveling wave and a particle of some momentum pv. However, it is now becoming clear that this analogy does not indicate wave-particle dualism, that is, not about the wave properties of the particle, but about the "particle-like" properties of the wave. These properties are especially characteristic of solitons as solitary structurally stable waves of elevation [15] so that their existence is beyond doubt. Thus, an ordinary (single) wave becomes a true quantum of radiation from the TIP position, which is explicitly discrete both in time and in space [16].

\section{Discussion of the Results}

The thermodynamic justification of the law of radiation proposed in series substantially supplements and simplifies its earlier conclusion [17]. This conclusion is quite consistent with the classical ideas about the wave nature of the radiation process and is not based on any quantum mechanical postulates or physical models such as a cavity with perfectly mirrored walls containing radiation with the properties of ideal gases. At the same time, it does not require the identification of radiant energy exchange with heat transfer and the existence of the thermal equilibrium between matter and radiation, which is clearly absent in outer space. He does not rely on model ideas about radiation as a variety of ideal gases with their characteristic temperature and entropy. In a word, the proposed proof of the Planck law of radiation is of the most general nature.

Another, no less important feature of the approach taken is the fact that the quantum nature of radiation is due to the nature of the wave process itself, the discreteness of which does not contradict classical concepts. Moreover, it is found that the universality of the value of $h$, called de Broglie "mysterious constant", is observed only for abstract blackbody cells with characteristic independence of the degree of blackness from frequency and a MaxwellBoltzmann distribution of amplitudes and pulses of oscillations between oscillators. It follows that there is no specific "quantum" physics with its own special laws there is a section of a single physics that studies wave processes.

From the stated positions, the erroneousness of a number of ideas underlying the foundation of quantum physics becomes apparent. In particular, the theory that the quantum energy of the oscillator energy $\varepsilon_{0}=h v$ is proportional to the frequency $n$ in the first, and not the second degree, contradicts the theory of waves, as follows from expression (1). The idea that the value of the Planck constant does not depend on the amplitude of the wave $A_{\mathrm{v}}$ is equally erroneous. Moreover, the idea of an increase in the energy of the oscillator with frequency is also erroneous. To verify this, it is enough to divide the wave energy density $\rho v$ defined by expression (1) by the number of oscillators The idea that the value of the Planck constant does not depend on the amplitude of the wave $A_{v}$ is equally erroneous.

Another contradiction is that the quantum energy increases unlimitedly with increasing frequency $\mathrm{n}$, and for high-frequency photons it can exceed the energy of the oscillator itself. A. Einstein first drew attention to this problem of the excess energy of a microwave quantum [18]. He showed by simple calculation that for a wavelength of $0.5 \mu \mathrm{m}$ at an absolute temperature $T=1700 \mathrm{~K}$, the quantum 
energy $\varepsilon_{\mathrm{o}}=h v$ в $6,510^{7}$ times higher than the energy of the oscillator itself!

It can also be shown that the wave energy density $\rho v$ determined by expression (1) decreases with frequency. To do this, it is enough to divide (1) by the number of oscillators $N_{v}=2 \pi v^{3} / c^{3}$ contained in a unit volume of the emitter and oscillating at a frequency $v$ :

$\varepsilon_{v}=\rho_{v} / N_{v}=\rho A_{v}^{2} c^{3} / 4 \pi v$

As follows from (12), at $A_{v}=$ const, the energy of one oscillator decreases with frequency due to the fact that $N_{v}$ grows faster with increasing frequency $v$ than their energy density $\rho_{v}$. This is precisely what prevents the so-called "violet catastrophe" arising from the law of Rayleigh radiation. Thus, the idea that the quantum of the radiation process is a wave discrete in space and time, and the quantum of action is its impulse, opens up the prospect of a convergence of classical and quantum physics [19].

\section{Acknowledgements:}

The author would like to thank IJoT publishing editor Dr. Ersin Sayar for his editing help.

\section{References:}

[1]. B. I. Spassky. History of Physics. V.2. M.: Higher school, 1977.(In Russian)

[2]. M. Planck. Über irreversible Strahlungsvorgänge. // Annalen der Physik, 306 (1) 1900. 69-122.

[3]. H. Poincare. L'hypothèse des quanta. // Revue Scientifique, 17 (4) 1912. 225-232.

[4]. M. Planck. Zur Geschichte der Auffindung des physikalischen Wirkungsquantums. Naturwissenschaften, 31 (14.15). 1943.153-159.

[5]. I. Prigogine. Etude Thermodynamique des Phenomenes Irreversibles. - Liege, 1947
[6]. De Groot S.R., Mazur P. Non-equilibrium Thermodynamics. - Amsterdam, 1962.

[7]. V.A. Etkin. Synthesis and new applications of theories of energy transfer and transformation. Diss. Dr. tech. Sciences.- M., MEI, 1998.

[8]. V.A. Etkin. Thermokinetics (Synthesis of Heat Engineering Theoretical Grounds) .- Haifa, 2010.

[9]. V.A. Etkin. Energodynamics (Thermodynamic Fundamentals of Synergetics) .- N.Y., 2011, 479 p.

[10]. F. Crawford. Waves. Berkeley Physics course. Vol. 3.- McGraw-Hill, 1968.

[11]. V.A. Etkin. On the potential and driving force of radiant heat transfer. // Bulletin of the House of Scientists of Haifa, 20 (2010). 2-6. (In Russian)

[12]. G.F. Muchnik, I. B. Rubashov. Methods of heat transfer theory. M.: Higher School, V.1-3, 1974. (In Russian)

[13]. L.D. Landau, E.M. Livshits, L.P. Pitaevsky. V.8. Physical kinetics. M.: Science, 1979. (In Russian)

[14]. L. de Broglie. The Reinterpretation of Wave Mechanics. // Foundations of Physics, 1 (1) .1970.6-18.

[15]. A. T Filippov. The many-faced soliton. Ed. 2.- M .: Nauka, 1990. (In Russian)

[16]. V.A. Etkin. Wave as a real quantum of radiation. // World scientific news, 66 (2017), 293-300.

[17]. V. Etkin. Rethinking Plank's radiation law. // Global Journal of Physics, 5 (2), 2017, 547-553

[18]. A. Einstein, L. Infeld. Evolution of Physics. Cambridge, 1938.

[19]. V.A. Etkin. Rethinking the fundamentals of quantum mechanics. // Problems of modern science and education, 12 (132) .2018, 6-14. DOI from $10.20861 /$ 2304-2338-2018-132-003. (In Russian) 